\title{
Die Akutsprechstunde in einer psychiatrischen Praxis
}

\author{
Ziel des Versorgungsstärkungsgesetzes (GKV-VSG) ist \\ unter anderem auch eine Verbesserung der psychothe- \\ rapeutischen Versorgung. Dass die DGPPN eine Akut- \\ sprechstunde für alle psychisch Kranken einer reinen \\ Psychotherapiesprechstunde vorzieht, wurde auf der \\ DGPPN-Jahrestagung 2014 deutlich. Zu dieser Thematik \\ erreichte uns ein Leserbrief von Heike Elspaß aus Alzey.
}

Mehrfach schon habe ich mich an verschiedene psychiatrisch/ psychotherapeutische Verbände, unter anderem auch an die KV in Mainz, gewendet und mitgeteilt, dass ich seit 2007 eine Akutsprechstunde in meiner Praxis anbiete. Gerade auch, als das GKV-VSG mit der Forderung der schnelleren und sektorenübergreifenden medizinischen Versorgung in die öffentliche Diskussion kam, versuchte ich mein Konzept der „Offenen Sprechstunde" Kollegen, aber auch Verbänden mitzuteilen. Leider gab es nie eine Resonanz! Ein Interview (Info Neurologie \& Psychiatrie 2015; 17 (1): 6 - 7) mit Professorin Sabine C. Herpertz „Die Akutsprechstunde wäre ein Schritt in die sektorenübergreifende Versorgung" (www.springermedizin.de/ 5551598) nehme ich zum Anlass, einen kurzen Abriss über die „Akutsprechstunde“ zu geben, wie ich sie in meiner Praxis durchführe: Ich sehe täglich von Montag bis Donnerstag zwischen 15 und 25 Patienten, die ohne Voranmeldung in die Praxis kommen können. Die Anmeldung erfolgt um 7.45 Uhr, die Behandlung erfolgt der Reihe nach. Ausgenommen sind hierbei natürlich medizinische Notfälle. Es handelt sich nicht um eine psychotherapeutische, sondern um eine psychiatrische Sprechstunde. Meine Hauptanliegen sind die medizinische Einschätzung, die medikamentöse Verordnung, die Koordination in Hinblick auf stationäre Aufenthalte, psychotherapeutische Weiterbehandlungen oder auch Behandlungen für andere Fachbereiche. Aufgrund der recht guten Zusammenarbeit ist ein interdisziplinäres Arbeiten in Alzey mit verschiedenen Fächern gut etabliert. Ebenso kann eine stationäre Aufnahme nach Rücksprache mit den Kliniken in Alzey, Mainz, Bad Kreuznach, Gleisweiler) zeitnah erfolgen. Häufig sind psychotherapeutische Gespräche (probatorische Sitzungen, die zeitlich auf je 30 Minuten gesplittet werden) ausreichend, um eine Stabilisierung der Patienten herbeizuführen. So entfällt das aufwändige Prozedere zur Aufnahme einer Richtlinienpsychotherapie. Falls jedoch eine Richtlinienpsychotherapie notwendig ist, kann die lange Wartezeit, die im Raum Alzey zwischen vier und acht Monaten beträgt, über regelmäßige Kontakte in der offenen Sprechstunde überbrückt werden. Nicht jeder psychisch kranke Mensch bedarf einer Richtlinienpsychotherapie, die psychiatrische Grundversorgung, vor allen Dingen die akute Grundversorgung stabilisiert viele meiner Patienten. Natürlich ist auch hier die Psychopharmakotherapie von großer Bedeutung, psychosoziale Interventionen, Hinzuziehen der Familie, Unterstützung bei Anträgen, Ämtern, Krankenkassen oder auch Rentenversicherungsanträge gehören ebenso zum alltäglichen Geschäft der offenen Sprechstunde. Über die Einrichtung der offenen Sprechstunde gibt es somit für die Patienten im Umkreis von Alzey viermal pro Woche die Möglichkeit einer direkten Kontaktaufnahme zum Facharzt für Psychiatrie und Psychotherapie. Ich halte diese Form von Behandlung für eine ausgezeichnete Möglichkeit, den Bedürfnissen der Patienten gerecht zu werden und die medizinische Versorgung, gerade im psychiatrischen Bereich deutlich zu verbessern.

\section{Heike Helena Elspaß}

Fachärztliche Praxis für Psychiatrie und Psychotherapie

Josselinstr. 39, 55232 Alzey

E-Mail: heike.h.elspass@t-online.de

\section{Ihre Meinung zählt!}

Was denken Sie über dieses Konzept der "offenen Sprechstunde“? Welche anderen Ansätze werden in anderen Praxen gelebt, die einer "Akutsprechstunde“ bereits nahe kommen? Schreiben sie uns: gunter.freese@springer.com.

Ein Modell einer spezialisierten Komplexversorgung bei Demenz, das bundesweit Schule macht, ist die Memo Clinic ${ }^{\circ}$ in Stralsund. Sie bietet eine teilstationäre spezialisierte Tagespflege, die auch Ärzte und Therapeuten einbindet. Dr. Stefanie Richter hat die Memo Clinic ${ }^{\bullet}$ als externe Wissenschaftlerin analysiert und stellt die Ergebnisse zusammen mit den Gründern, Olaf H. Waehnke und Dr. Ronald R. Zabel, vor. Die als Franchise-Modell angelegte Memo Clinic ${ }^{\circ}$ (www.memoclinic. de) mit bisher drei Standorten ist ein in der Praxis etabliertes Versorgungsmodell für Menschen mit Demenz, das unterschiedliche Akteure, ambulante und teilstationäre Strukturen sowie Betreutes Wohnen integriert. Es leistet fachübergreifende Versorgung in Form interprofessioneller und intersektoraler Kooperationen. 